\title{
Effective parameters for stimulation of dissociated cultures using multi-electrode arrays
}

\author{
Daniel A. Wagenaar ${ }^{\mathrm{a}, *}$, Jerome Pine ${ }^{\mathrm{a}}$, Steve M. Potter ${ }^{\mathrm{b}}$

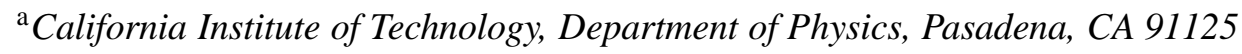 \\ ${ }^{\mathrm{b}}$ Georgia Institute of Technology, Department of Biomedical Engineering, Atlanta, \\ GA 30332
}

\begin{abstract}
Electrical stimulation through multi-electrode arrays is used to evoke activity in dissociated cultures of cortical neurons. We study the efficacies of a variety of pulse shapes under voltage- as well as current-control, and determine useful parameter ranges that optimize efficacy while preventing damage through electrochemistry. For any pulse shape, stimulation is found to be mediated by negative currents. We find that positive-then-negative biphasic voltage-controlled pulses are more effective than any of the other pulse shapes tested, when compared at the same peak voltage. These results suggest that voltage-control, with its inherent control over limiting electrochemistry, may be advantageous in a wide variety of stimulation scenarios, possibly extending to in-vivo experiments.
\end{abstract}

Key words: Electrical stimulation; dissociated culture; multi-electrode array; MEA; stimulation parameters.

\section{Introduction}

Multi-electrode arrays (MEAs) (Thomas et al., 1972; Gross, 1979; Pine, 1980; Potter, 2001; Heuschkel et al., 2002) have been used to record from a wide variety of neuronal preparations. Electrical stimulation through MEAs has been used to elicit spiking activity in dissociated cultures (Regehr et al., 1988; Jimbo and Kawana, 1992; Gross et al., 1993; Maher et al., 1999b; Jimbo et al., 1999), as well as brain slices (Heck, 1995; Egert et al., 1998; Novak and Wheeler, 1988; Echevarria and Albus, 2000; Harsch and Robinson, 2000; Tscherter et al., 2001) and isolated retina (Branner and Normann, 2000; Grumet et al., 2000). MEAs and related technology for multi-site extracellular stimulation and

\footnotetext{
* Corresponding author. Caltech 103-33, Pasadena, CA 91125. Tel. (626) 395-6787.

Email address: wagenaar@caltech.edu (Daniel A. Wagenaar).
} 
recording, such as silicon probes (Wise and Angell, 1975; Bai and Wise, 2001) and multiwire probes (Nicolelis et al. (1998)) have gained popularity because the relatively noninvasive nature of the technology allows for long-term interaction with healthy cells, and because they scale well to a large number of recording and stimulation channels.

When designing stimulation paradigms, researchers have to make many choices even after they have decided on electrode material: whether to use bipolar stimuli (between two electrodes) or monopolar stimuli (between one electrode and a large and usually distant ground electrode), whether to use voltage or current control, what pulse shape to use (monophasic, biphasic, perhaps even multiphasic or asymmetric). Compromises have to be found between effi cacy of stimuli, harm to electrodes or cells, and stimulation artifacts that hamper recording of responses. For long-term experiments, it is crucial to prevent damaging electrodes and killing cells. Cell damage can result from high charge injection or high charge densities (McCreery et al., 1990), but our MEA electrodes cannot inject dangerous amounts of charge before exceeding electrolysis limits (Weiland et al., 2002). Electrolysis, which starts to play a role when electrode voltages exceed about one volt directly damages electrodes, and is also harmful to cells. (This harm can be much reduced by employing charge-balanced stimuli, making such stimuli preferable for long-term or in-vivo work when large voltages cannot be avoided (Lilly et al., 1955; Shepherd et al., 1991).) A secondary constraint can be the width of stimulus pulses: Since recording is generally impossible for the duration of the stimulus pulse, short pulses are often desirable.

Here, we study electrical stimuli intended to evoke activity in dissociated cortical cultures on MEAs, with the aim of establishing robust two-way communication between such cultures and a computer system. Knowing a set of stimuli that are reliably and consistently effective is essential for long-term experiments on the development of functional networks, as well as for research on learning in-vitro (DeMarse et al., 2001; Shahaf and Marom, 2001). While the electrical properties of MEA electrodes have been described in the literature (Kovacs, 1994; McAdams et al., 1995; Buitenweg et al., 1998; McIntyre and Grill, 2001), the published knowledge base on what kinds of stimuli are most effective at evoking responses is remarkably slim. A full quantitative understanding would require a detailed model of the electric fi elds that current pulses induce along axonal and somatic membranes, but in high density cultures, the arrangement of neurons and glia is too complex to construct such a model. In this paper we hope to provide new practical information by identifying a range of stimuli that are effective, unharmful, and produce minimal artifacts. The results in this paper were obtained from dense neocortical cultures grown on MEAs with $30 \mu \mathrm{m}$ titanium nitride electrodes. Qualitatively, the results should extend to other dissociated neuronal cultures, and to electrodes of different sizes and construction. 


\section{Methods}

\subsection{Cell culture}

Neocortex was dissected from rat embryos (E18) under sterile conditions. Cortices were cut into pieces of about $1 \mathrm{~mm}^{3}$, prior to dissociation using papain and trituration. Cells were plated at 5000 cells $/ \mathrm{mm}^{2}$ on multi-electrode arrays (MultiChannel Systems, Reutlingen, Germany) coated with poly-ethylene-imine (PEI) and laminin. Cultures were maintained for 2-3 weeks prior to recording, in a medium adapted from Jimbo et al. (1999): High glucose DMEM (Irvine Scientifi c cat. no. 9024) with 10\% Horse Serum (HyClone), $0.5 \mathrm{mM}$ GlutaMax (Gibco cat. no. 5050.061), $1 \mathrm{mM}$ sodium pyruvate, and $2.5 \mu \mathrm{g} / \mathrm{mL}$ insulin. To prevent evaporation and to stop infection, culture dishes were sealed using Teflon membranes (Potter and DeMarse, 2001). Cultures were kept in an incubator at $35{ }^{\circ} \mathrm{C}, 65 \%$ R.H., $5 \% \mathrm{CO}_{2}$, and $9 \% \mathrm{O}_{2}$. All experiments were performed inside this incubator, guaranteeing stability of environmental conditions. (At $65 \%$ relative humidity, our incubator is safe for electronics.)

\subsection{Pharmacology}

For blocking glutamatergic synaptic transmission, we replaced the cell culture medium with a medium pre-mixed with $800 \mu \mathrm{M}$ D(-)-2-Amino-5-phosphonopentanoic acid (AP5, Sigma A-169), an NMDA-channel blocker, and $80 \mu \mathrm{M}$ 6-cyano-7-nitroquinoxaline-2,3dione disodium salt (CNQX, Sigma C-239), an AMPA-channel blocker. For blocking all activity, we used a medium pre-mixed with $1 \mu \mathrm{M}$ tetrodotoxin (TTX, Sigma T-5651). Prior to either kind of blocker experiment, we obtained a baseline by replacing all of the growing medium with $1 \mathrm{~mL}$ of fresh medium and recording responses to voltage stimulation after allowing the culture to equilibrate for 30 minutes. We then prepared $1 \mathrm{~mL}$ of medium with either AP5 and CNQX or with TTX, and replaced the culture medium with this mixture, again recording after 30 minutes of equilibration. After that, we washed out the blockers by four full medium replacements spread out over fi ve minutes, and recorded a fi nal run through the stimulus set to confi rm return to baseline conditions.

\subsection{Electrodes and recording system}

We use glass MEAs with $30 \mu \mathrm{m}$ diameter titanium nitride electrodes and a silicon nitride insulation layer (MultiChannel Systems, Reutlingen, Germany). The electrodes have a rough surface which increases their capacitance for a given electrode diameter. Each MEA has 59 such electrodes laid out in a rectangular grid with $200 \mu \mathrm{m}$ interelectrode spacing. Signals were amplifi ed using an MEA60 preamplifi er, and digitized using an MC_Card PCI board (both MultiChannel Systems). Data acquisition was controlled using 
MeaBench software (D. A. Wagenaar, http://www.its.caltech.edu/ wagenaar/meabench). MeaBench was also used for on-line data visualisation and processing: Stimulation artifacts were suppressed on-line using the SALPA algorithm (Wagenaar and Potter, 2002), after which spikes were detected by thresholding at $5 \times$ RMS noise. Candidate spikes were validated using a simple shape-based criterion (P. P. Mitra, personal communication).

\subsection{Stimulation system}

\subsubsection{Current pulses}

We produced current-controlled stimuli by passing the output of a PCI-6216 digital-toanalog convertor (DAC) card (AdLink Technologies, Taiwan) through the voltage-tocurrent convertor depicted in Figure 1A (Horowitz and Hill, 1996). This circuit allows us to monitor the voltage generated by the current pulses. We tested both positive ("an-

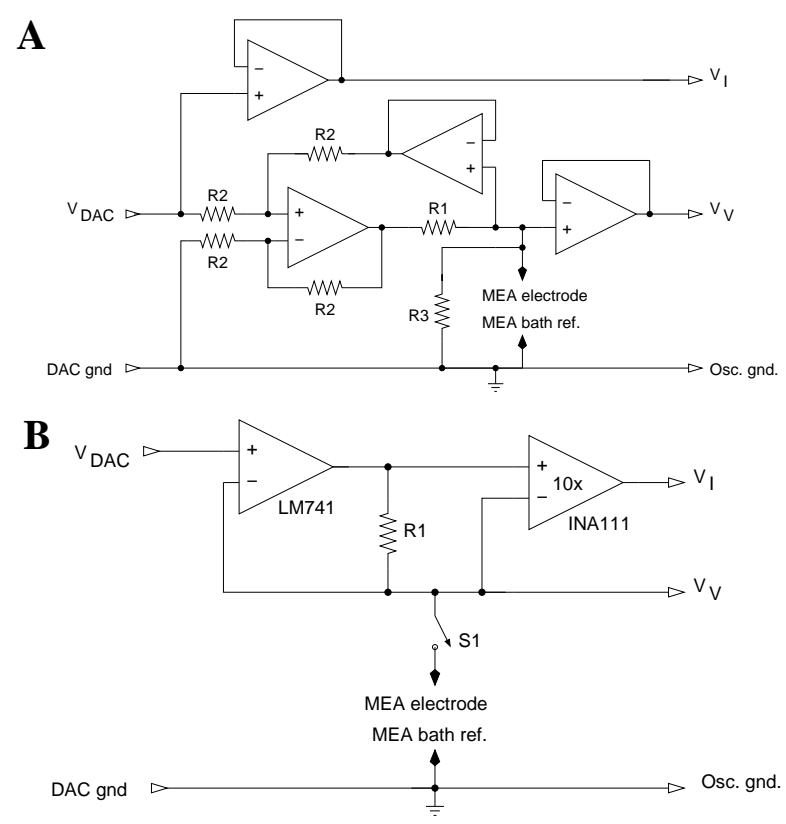

Figure 1. Stimulation hardware. A. Circuit for current-controlled stimulation. $V_{\mathrm{DAC}}$ is converted to a current $I=V_{\mathrm{DAC}} / R_{1}$ through the resistor $R_{1}=150 \mathrm{k} \Omega . R_{3}=6.8 \mathrm{M} \Omega$ acts as a shunt to prevent runaway electrode voltages. (Electrode impedances are $50-200 \mathrm{k} \Omega$ at $1 \mathrm{kHz}$ ). $R_{2}=150 \mathrm{k} \Omega$. Opamps are $\frac{1}{4} \mathrm{LM} 348$. The terminals $V_{\mathrm{V}}$ and $V_{\mathrm{I}}$ are used to monitor the electrode voltage and the driving current (multiplied by $R_{1}$ ). B. Circuit for voltage-controlled stimulation. The MEA electrode is driven at the voltage $V_{\text {DAC. }}$. The voltage across $R_{1}=5.0 \mathrm{k} \Omega$, amplified and measured at $V_{\mathrm{I}}$, directly reflects the current passing through the electrode. The electronic switch $S_{1}$ can be used to effectively disconnect the stimulator from the MEA, allowing neuronal signals to be recorded through the stimulated electrode shortly after stimulation. This circuit is part of a device for delivering stimuli to any electrode in an MEA with rapid switching between channels (Wagenaar and Potter, 2004). 
odic") and negative ("cathodic") monophasic currents of various strengths $(1 \mu \mathrm{A}-20 \mu \mathrm{A})$ and duration $(10 \mu \mathrm{s}-1000 \mu \mathrm{s})$ on 55 electrodes from 5 different MEAs. Additionally, we tested biphasic (positive-then-negative or negative-then-positive) current pulses on six electrodes from two MEAs. (The stimulus repertoire is summarized in Table 1.) Electrodes were randomly selected, subject to the constraint that only electrodes were chosen that recorded activity during spontaneously occurring culture-wide barrages of action potentials. All stimuli were relative to a large electrode embedded in the MEA substrate which also served as a reference electrode for recording responses. Each pulse type was presented 50 times to each stimulation electrode, with one second between trials. Presentation order was randomized. Stimulation was fully automated using custom software controlling the hardware through real-time Linux. Responses to stimuli were measured using all non-stimulated electrodes, as described below.

Table 1. Summary of current-controlled pulses tested.

\begin{tabular}{lcc}
\hline Pulse shape & Amplitudes $(\mu \mathrm{A})$ & Pulse widths $(\mu \mathrm{s})$ \\
\hline Monophasic, negative & $1-10$ & $20-1280$ \\
Monophasic, positive & $1-10$ & $20-1280$ \\
Biphasic, negative-first & 5,10 & 50,200 \\
Biphasic, positive-first & 5,10 & 50,200 \\
\hline
\end{tabular}

\subsubsection{Voltage pulses}

Voltage-controlled stimuli were generated using either one channel from the Adlink DAC used for current stimulation, or using a custom DAC based on a TLC-7628 (Texas Instruments). To monitor the current resulting from the stimulus, we used the circuit depicted in Figure 1B. We tested biphasic (positive-then-negative and negative-then-positive) pulses of various amplitudes $(100 \mathrm{mV}-1000 \mathrm{mV})$ and duration $(100 \mu \mathrm{s}-800 \mu$ s per phase $)$ on 45 electrodes from 5 MEAs. Additionally, we tested monophasic (positive and negative) pulses on six electrodes from two MEAs. Finally, we tested "unterminated" pulses, that is, biphasic voltage pulses with the fi nal phase not ended by driving the voltage back to zero but by opening the stimulation switch ('S1' in circuit diagram), thus cutting the driving current. (The stimulus repertoire is summarized in Table 2.) As before, a large substrate electrode doubled as return and reference.

\section{Results}

All electrodes tested could be used to evoke responses, given suffi ciently strong stimuli (Figure 2). Responses to individual stimuli could be differentiated into three parts: 
Table 2. Summary of voltage-controlled pulses tested.

\begin{tabular}{lcc}
\hline Pulse shape & Amplitudes $(\mathrm{mV})$ & Pulse widths $(\mu \mathrm{s})$ \\
\hline Monophasic, negative & $100-1000$ & 400 \\
Monophasic, positive & $100-1000$ & 400 \\
Biphasic, negative-first & $100-1000$ & $100-900$ \\
Biphasic, positive-first & $100-1000$ & $100-900$ \\
'Unterminated' (see text) & 500 & $100-400$ \\
\hline
\end{tabular}

Direct responses - Any response that does not depend on glutamatergic synapses. These occur in the fi rst 10-20 ms post-stimulus, have less than $0.25 \mathrm{~ms}$ temporal jitter and can be close to $100 \%$ reliable (i.e. observed in close to $100 \%$ of trials). Direct responses most likely result from antidromic excitation through an axon near the stimulating electrode, though in principle they could result from a single axon passing by both the stimulation and the recording electrode, or from a cell with soma near the stimulation site and an axon passing the recording electrode. We did not study the potential role of gap junctions, but others have reported that it is minimal (Nakanishi and Kukita, 1998).

Early post-synaptic spikes - Responses that do depend on glutamatergic synapses, occurring between $5 \mathrm{~ms}$ and $50 \mathrm{~ms}$ post-stimulus. Their temporal precision varies around $2 \mathrm{~ms}$, and their reliability ranges up to $30 \%$, though values around $10 \%$ are more common.

Culture-wide barrages - In some fraction of trials, stimulation results in a barrage of activity spreading over the entire recording array. Depending on the electrode stimulated, such barrages can initiate immediately after stimulation, or at latencies exceeding $100 \mathrm{~ms}$. In most cultures, barrages cannot be evoked more than once every $2-5 \mathrm{~s}$.

For the present report, the fi rst part is most important: the direct responses indicate how many cells are immediately stimulated by a given pulse. Using results from ten stimulation electrodes in two cultures, we found that blocking glutamatergic synapses inhibited all responses except for the very precisely timed ones (Figure 3). Thus, we found that direct responses are distinguishable from post-synaptic responses by their extremely reproducible timing: their temporal jitter is always less than $0.25 \mathrm{~ms}$, compared to more than $1 \mathrm{~ms}$ for most post-synaptic responses. Based on this characteristic, we could distinguish direct responses even in medium without synapse blockers. We confi rmed their biological origin by application of the sodium-channel blocker TTX, which reversibly abolished all spikes (data not shown).

Direct responses were observed even at electrodes distant from the stimulation site, but somewhat more frequently nearby the stimulus. Direct responses recorded at different electrodes were not correlated across trials of a given stimulus strength, indicating that 


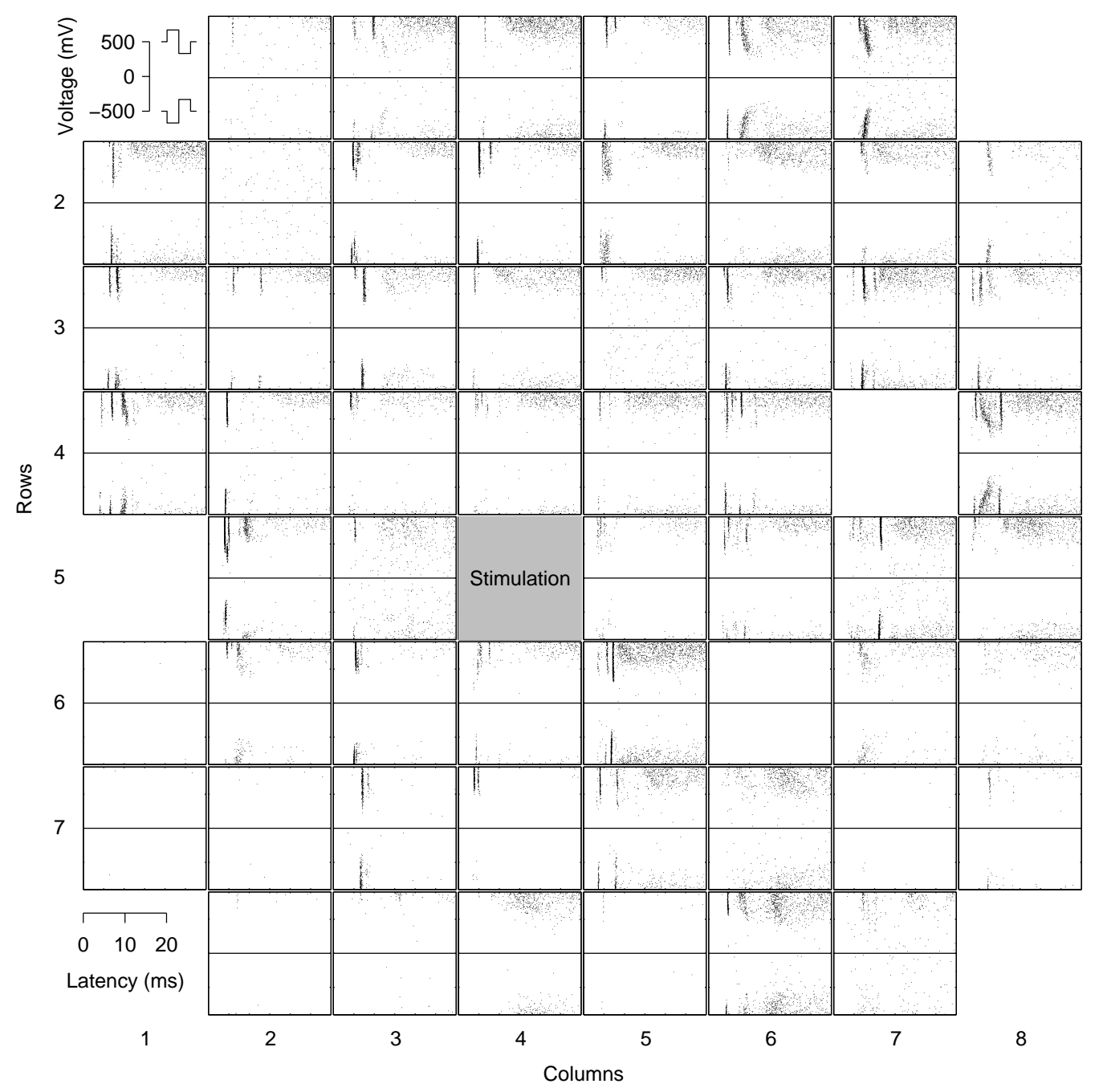

Figure 2. Array-wide responses to biphasic voltage pulses on the marked electrode during $30 \mathrm{~ms}$ after stimulation. Graphs are laid out following the physical geometry of the MEA - a rectangular array with $200 \mu \mathrm{m}$ inter-electrode spacing. Each graph shows responses vs time from one electrode. Within each graph, positive-then-negative stimuli are shown in the top half, with stronger stimuli towards the top, while negative-then-positive stimuli are shown in the bottom half, with stronger stimuli towards the bottom. Each dot represents a single spike. These data were accumulated over 50 trials at each of 36 voltage levels between -900 and $+900 \mathrm{mV}$, with spikes from each trial in horizontal lines. Direct responses appear as very sharp vertical clusters, post-synaptic responses as larger clouds. This representation does not show the occasional occurrence of barrages.

those responses are due to different axons independently stimulated by the pulse, rather than due to a single axon passing by several recording electrodes. 


\subsection{Current pulses}

Many researchers prefer current pulses on theoretical grounds, because the electric fi eld and potential near the electrode can be directly calculated (Maher et al., 1999a). Thus the effects of current pulse stimulation are thought to be better understood than the effects of voltage pulse stimulation, for which the (current-induced) electric fi eld depends on the complex impedance of the stimulation electrode. In reality, a signifi cant fraction of current applied to the electrode may be lost to leakage through the insulation of the electrode leads (see below under Voltage pulses), reducing the reliability of such calculations.

For a typical new electrode with low leakage currents, $10 \mu \mathrm{A}$ current pulses of $50 \mu \mathrm{s}$ duration resulted in an electrode voltage of about $1 \mathrm{~V}$ (Figure 4A). Robust responses were observed after stimulating with negative currents (Figure 5). Positive pulses never resulted in any response. Increasing amplitude or duration of negative currents improved the chance of eliciting responses, as expected. Very short (50 $\mu \mathrm{s})$ current pulses evoked as many spikes as long pulses with the same charge transfer, a surprising result, given that the time-constant for the opening of sodium channels is about $0.8 \mathrm{~ms}$ (Sigworth and Neher, 1980). For long pulse widths ( $200 \mu \mathrm{s})$, biphasic pulses were not signifi cantly more or less effective than monophasic negative pulses (Figure 5A). For short pulse widths (50 $\mu \mathrm{s})$, biphasic pulses were less effective than monophasic negative pulses, presumably because the two phases partially cancel each other in the temporal integration performed by the target neuron's population of sodium channels (data not shown).

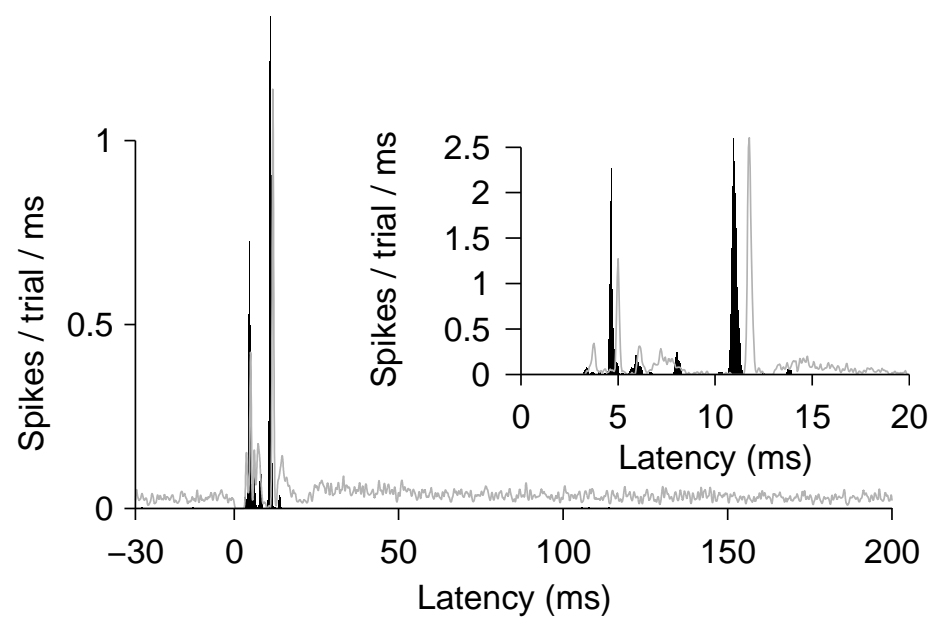

Figure 3. Firing rate on one electrode as a function of latency after stimulus. Baseline (grey) and in the presence of AP5 and CNQX (black). Inset shows detail at early latency. Data was pooled from 475 trials. Synaptic blockers abolished all activity except for the precisely timed spikes ('direct responses'). Note the shift in latency of the direct responses. This is likely due to more sodium channels being ready to open when synapses are blocked — in baseline conditions, a certain fraction of sodium channels is always in the inactivated state due to ongoing spiking activity. The small peaks near 6 and $8 \mathrm{~ms}$ latency are due to events near the detection threshold. 
A

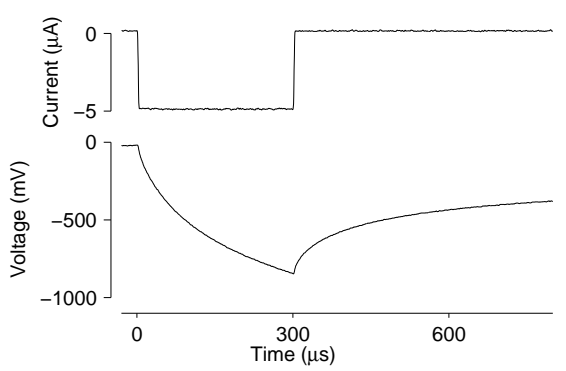

B

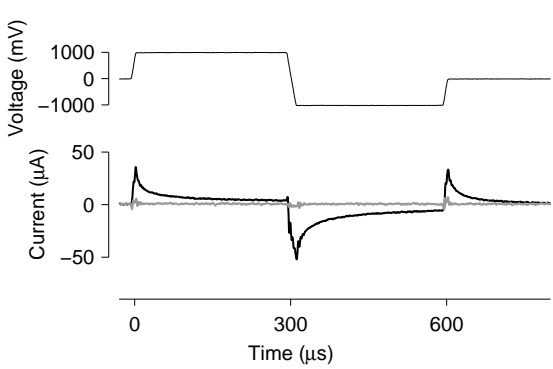

C

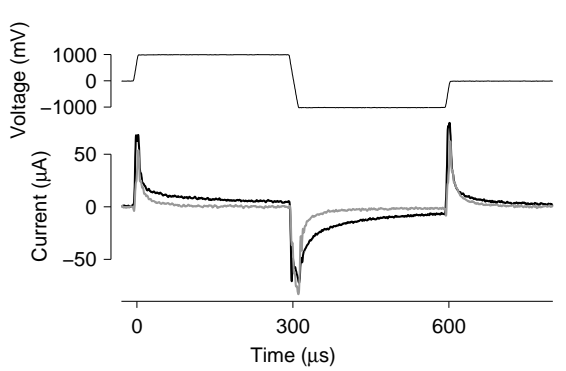

Figure 4. Stimulation waveforms. A. Current-controlled stimulation: Driving current (top) and the resulting voltage (bottom). The discharge after cessation of driving current is through a shunt resistor (see Figure 1A). Waveforms were recorded using a Tektronix TDS2014 digital storage oscilloscope connected to the $V_{\mathrm{V}}$ and $V_{\mathrm{I}}$ terminals in Figure 1A. B. Voltage-controlled stimulation: a typical driving voltage waveform (top) and the corresponding current response (bottom) for an electrode on an MEA that has been plated just once. We distinguish true electrode currents (black) from leakage currents (grey) (see text). Currents due to stray capacitance have been subtracted out. Waveforms were recorded from the $V_{\mathrm{V}}$ and $V_{\mathrm{I}}$ terminals in Figure 1B through 10x probes. C. Voltage-controlled stimulation through an electrode on an older MEA, showing substantial leakage current, presumably through damaged insulation.

\subsection{Voltage pulses}

Unfortunately, current-controlled stimulation has several practical drawbacks: good current sources are more complex than voltage sources, but more importantly, current pulses can cause high electrode voltages, which may harm cultures or damage electrodes through electrochemistry. For these reasons, most stimulation experiments on MEAs in our labs are actually performed under voltage-control.

Positive-then-negative biphasic voltage stimuli were very effective at eliciting action po- 
tentials, while suffi ciently strong negative-then-positive stimuli also elicited action potentials (Figure 6). Responses were recorded across the entire array, although electrodes close to the stimulation electrode were slightly more likely to record responses. The voltage dependence of responses was the same for recording electrodes near to and far from the stimulation electrode (data not shown). Monophasic positive pulses did not elicit many responses, despite the fact that the downward transient at the end of the pulse corresponds to a strong negative current. Conversely, monophasic negative pulses were effective, but signifi cantly less so than biphasic pulses.

A

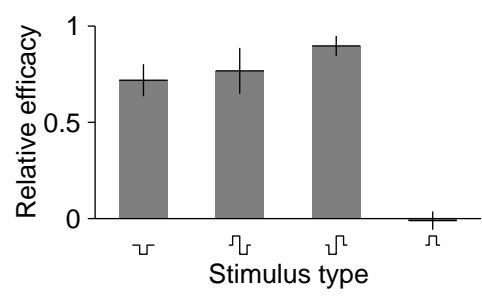

B

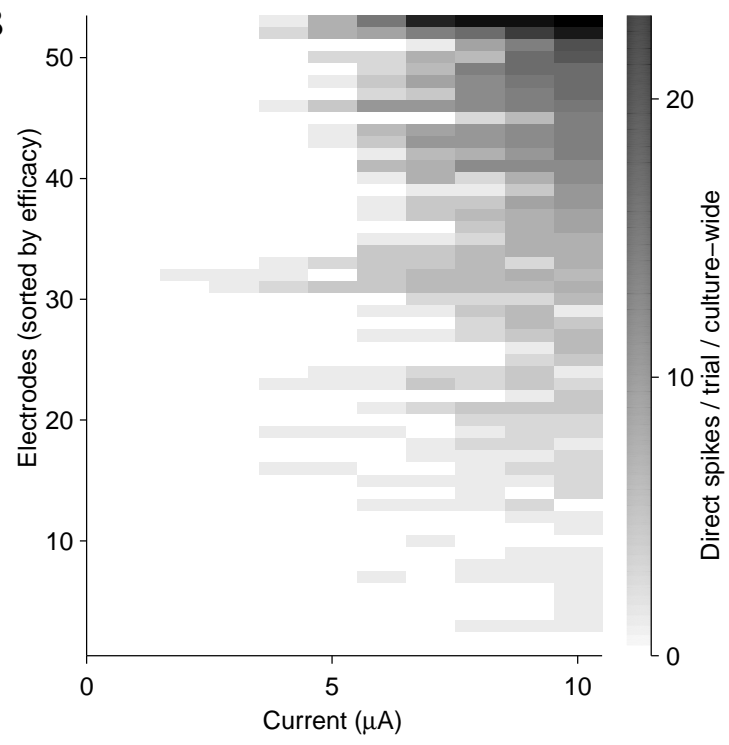

C

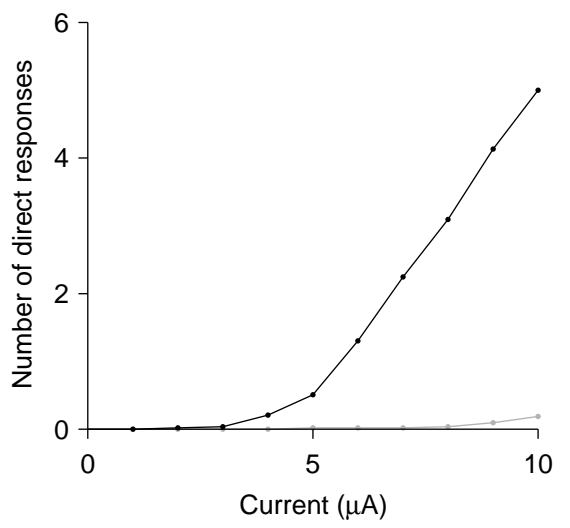

D

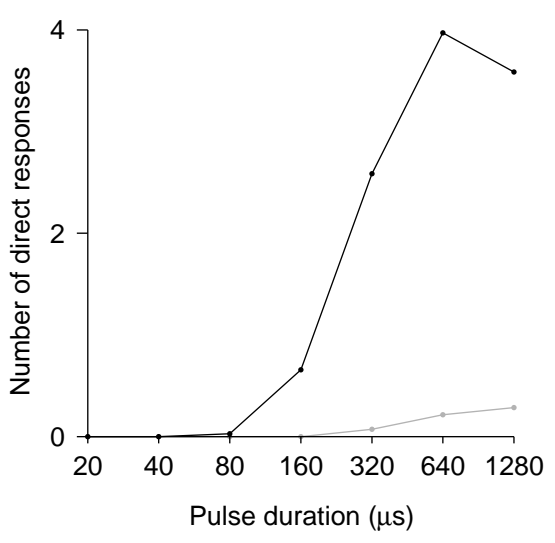

Figure 5. Responses to current stimulation. A. Comparison of pulse shape efficacies: We measured the per-trial-average number of spikes recorded array-wide in the first $20 \mathrm{~ms}$ after stimulation, for various pulse shapes (left to right: monophasic negative; biphasic positive-then-negative; biphasic negative-then-positive; monophasic positive), with amplitude $5 \mu \mathrm{A}$ and duration $200 \mu$ s per phase. Baseline firing rates were subtracted, and results were normalized by the value for the most effective pulse. Shown are averages and SEM $(N=6)$. B. Number of direct responses (across the array) recorded after monophasic negative current pulses of $50 \mu$ s duration and varying amplitudes. Based on 55 electrodes in 5 MEAs. C. Average across electrodes of data in $B$ (black). Positive pulses (grey) did not excite spikes. D. Average number of direct responses after negative (black) and positive (grey) current pulses of $1 \mu \mathrm{A}$ amplitude and varying durations. 
At fi xed duration, response reliability generally increased with increasing stimulus amplitude, though about $20 \%$ of precise responses decreased in reliability above a certain point (Figure 7A). At fi xed stimulus amplitude, the number of responses did not depend strongly on pulse duration, once the duration is above threshold. For short positive-then-negative pulses, the effi cacy could be signifi cantly improved by leaving the negative phase unterminated (cutting the stimulation current rather than explicitly driving the voltage back to

A

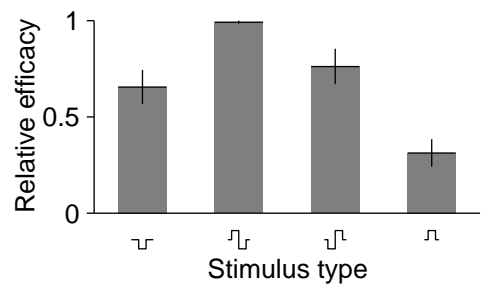

B

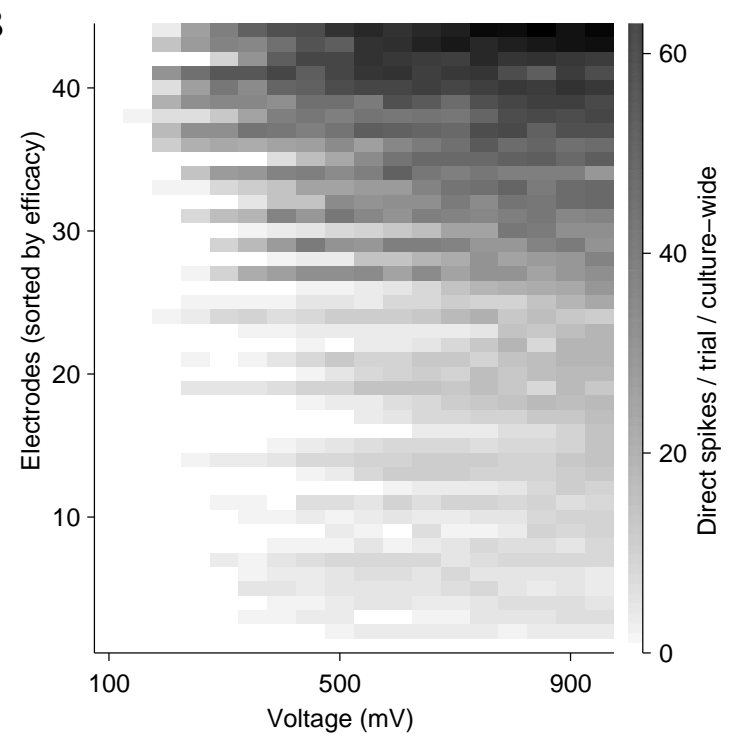

C

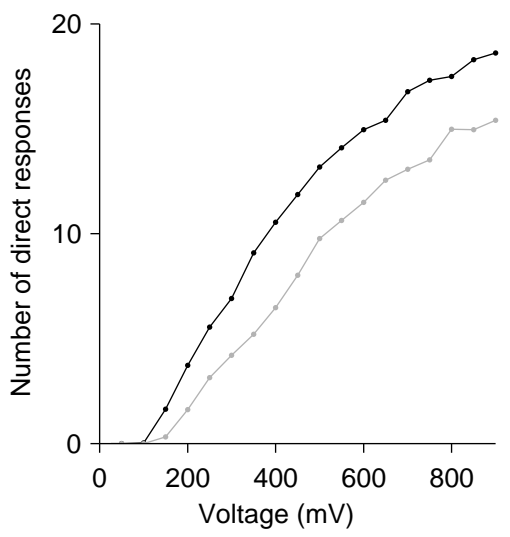

D

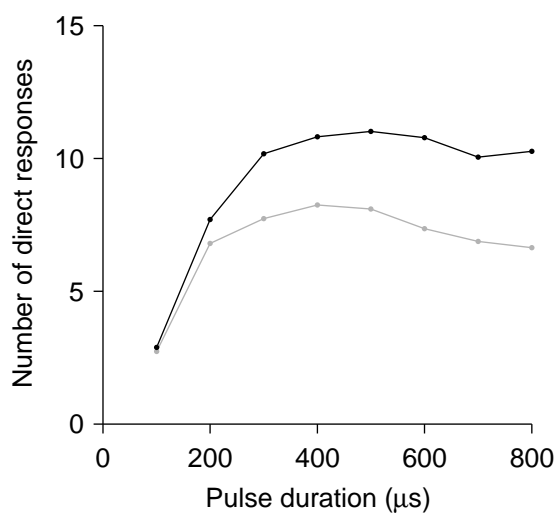

Figure 6. Responses to voltage stimulation. A. Comparison of pulse shape efficacies: We measured the per-trial-average number of spikes recorded array-wide in the first $20 \mathrm{~ms}$ after stimulation, for various pulse shapes (left to right: monophasic negative; biphasic positive-then-negative; biphasic negative-then-positive; monophasic positive), with amplitude $500 \mathrm{mV}$ and duration $400 \mu \mathrm{s}$ per phase. Baseline firing rates have been subtracted, and results were normalized by the value for the most effective pulse. Shown are averages $(N=6)$ and SEM. B. Number of direct responses (across the array) recorded after biphasic positive-then-negative voltage pulses of $400 \mu$ s duration and varying amplitudes. Based on 45 stimulation electrodes in 5 MEAs. C. Average across electrodes of data in B (black). Responses to negative-then-postive stimulation are slightly less strong (grey). D. Average number of direct responses after positive-then-negative (black) and negative-then-positive (grey) voltage pulses of $500 \mathrm{mV}$ amplitude and varying durations (same 45 electrodes). Note that very long pulses result in fewer recorded responses, because some early responses get obscured by stimulation artifacts. 
baseline). This effect was much weaker for longer pulses.

To determine which part of a biphasic voltage pulse gives rise to the responses, we fi tted the latency of well-isolated direct spikes as a linear function of per-phase-width of the stimuli. If the spikes are evoked by the initial transient, this function should have zero slope; if the spikes are evoked by the second transient, the slope should be one; and if the fi nal transient is responsible, the slope should be two. (Slopes between zero and one indicate that the first constant phase, rather than the transient, is responsible, while slopes between one and two indicate that the second phase is responsible.) We found that most direct spikes are time-locked to the (fi rst) downward transient in the voltage waveform: for positive-then-negative stimuli, the latency from stimulus onset typically increases directly with pulse width (median slope of latency vs phase width is $1.01 \mu \mathrm{s} / \mu \mathrm{s}$ ), while for negative-then-positive stimuli, the latency doesn't change much (median slope is $0.23 \mu \mathrm{s} / \mu \mathrm{s}$ ). This confi rms the observation that only negative current pulses are effective stimuli.

\subsection{Number and reliability of responding cells}

In principle, when changing a parameter increases the number of direct spikes observed in the response, this can be due to either of two mechanisms: more neurons can become directly activated by the stimulus, or the activation of previously activated neurons can become more reliable. We isolated direct responses occurring in the first $30 \mathrm{~ms}$ poststimulation using a clustering algorithm (see Appendix) that identifi ed sharp peaks in the post-stimulus time histogram (PSTH). From this, we calculated the 'reliability' of a direct response (at a given stimulus strength) as the fraction of trials in which it is observed. We defi ned the 'relative reliability' of a direct response as the absolute reliability of that response divided by the peak reliability of that response. Aligning the relative-reliability curves of many direct responses to their threshold value (point of 50\% reliability), we found that with increasing stimulus strength, individual direct responses were initiated above sharply defi ned thresholds: a change of $10 \%$ in pulse amplitude could make the difference between no response and near-maximal response (Figure 7). This held for both voltage-controlled and current-controlled stimuli, suggesting that the increase in number of recorded spikes is due to an increased number of cells fi ring action potentials in direct response to the stimulus, and not due to an increase in the reliability of the fi ring of any individual cell.

\subsection{Voltage vs current-control: electrode and leakage currents}

For comparison of current- and voltage-controlled stimuli, it is important to distinguish between currents flowing through the electrode, and currents lost through leakage or arising purely from stray capacitance in cabling. (Stray capacitance is especially signifi cant for voltage-controlled pulses, because sharp voltage transients cause strong capacitive 
A

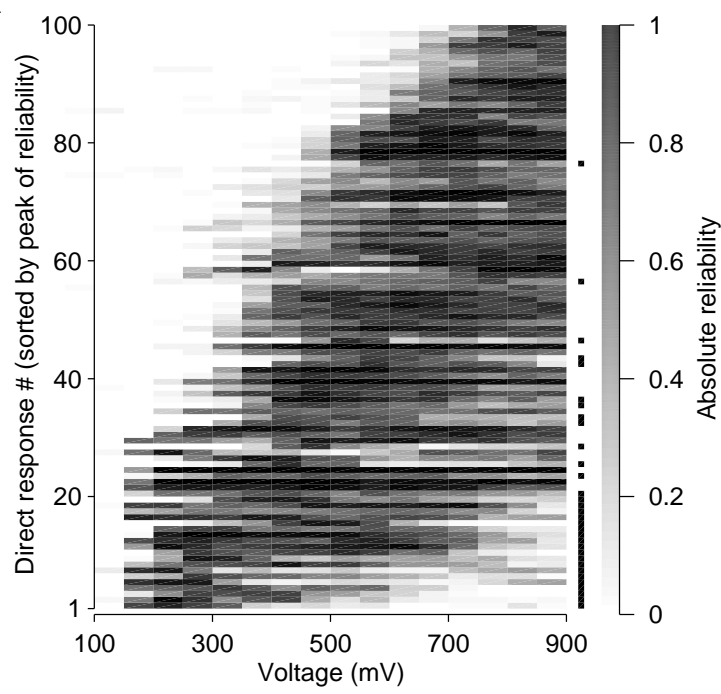

B

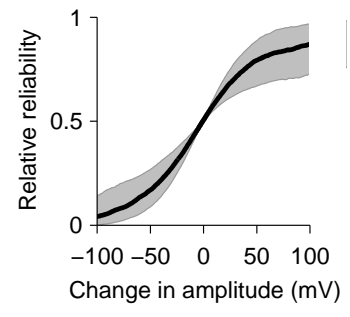

C

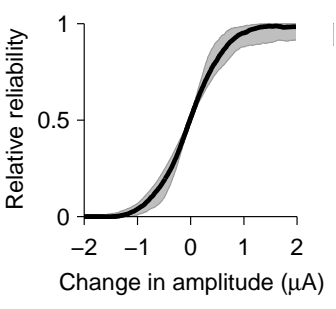

Figure 7. A. Reliability of a random selection of 100 direct responses, sorted by the stimulation voltage at which their reliability peaks. While most direct responses increase in reliability with increasing voltage, a subset only occurs in a voltage range (indicated by black marks on right). B. Relative reliability (see text) of direct responses evoked by voltage-controlled pulses near their threshold. Notice the sharpness of the onset. Curve is average of 757 direct responses, shaded area indicates quartiles. C. Relative reliability of direct responses evoked by current-controlled pulses near their threshold. Curve is average of 362 direct responses, shaded area indicates quartiles.

currents.) We measured the leakage currents explicitly by applying voltage pulses after fencing off the electrode area of three MEAs using the end of a wide-bore pipette tip held in place with vaseline. Thus we could flood the rest of the MEA dish and measure most of the lead-to-medium conductance as well as stray capacitance in isolation from electrode conductance. Stray capacitance, at $450 \mathrm{pF}$, was responsible for most of the current peak during voltage transients. While we found leakage currents to be insignifi cant (less than $5 \%$ of total current) for brand new MEAs, they could constitute up to $30 \%$ of the total current path in 6-month-old MEAs that had been plated several times (see Figure 6B). Upon physical inspection, such older dishes are often found to exhibit microscopic cracks in the insulation layer.

In all but one MEA tested, voltage pulses were much more effective than current pulses that reached the same peak voltage. We defi ned the 'effi cacy' of a stimulus as the number of spikes that stimulus evoked on average, normalized by the number of spikes evoked by the most effective stimulus in the repertoire. To compare effi cacies of voltage and currentcontrolled stimuli, we measured the peak electrode voltage reached during current-controlled stimulation, and plotted the experimental effi cacies as a function of this peak voltage (Figure 8). One MEA in our data set exhibited anomalously strong responses to current stimuli. We found that this MEA had large electrode conductance, but also exceptionally large leakage (as shown in Figure 4C), presumably due to damaged insulation. 


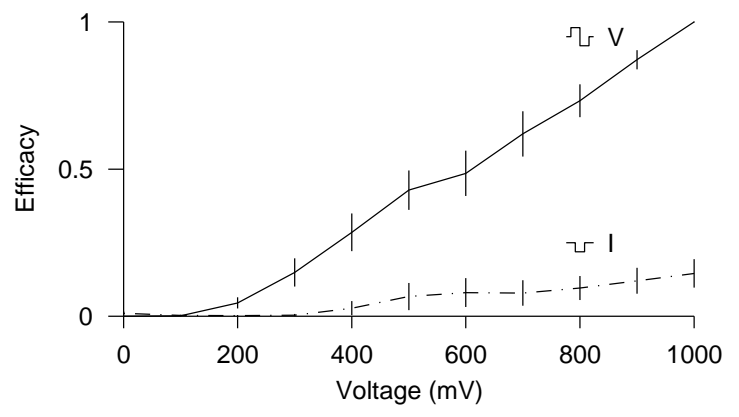

Figure 8. Efficacy (see text) of positive-then-negative voltage-controlled pulses of $400 \mu$ s per-phase (solid) and monophasic negative current pulses of $50 \mu$ s duration for which the resulting voltage was measured (dashed). The voltage-controlled pulses are significantly more effective. (Average and SEM, $N=6$.)

\section{Discussion}

From the great variety of possible stimulation pulse shapes that can be applied to MEA electrodes, we studied eight important families: monophasic and biphasic rectangular current pulses of either polarity, and monophasic and biphasic rectangular voltage pulses of either polarity. We found that the effi cacy of stimuli in any of these families can be attributed to the generation of negative electrode currents.

To explain why negative current pulses are effective stimuli while positive currents are not, others have modeled the intracellular currents resulting from extracellular fi elds. They found that stimulation effi cacy follows the second spatial derivative of the potential along the length of an axon (Rattay, 1999; McIntyre and Grill, 1999). For stimulation of cell bodies or unmyelinated axons very near electrodes, we can consider the following simpler equivalent picture: Negative current pulses lower the potential of the medium near the electrode. This can evoke an action potential if the pulse is long enough to signifi cantly charge the local membrane capacitance, thus depolarizing that membrane. A simple model suggests that the relevant time constant for stimulation of axons that traverse an electrode is of the order of tens of microseconds, thus explaining how even the very short current pulses resulting from voltage-controlled stimulation can be effective stimuli. This picture also explains why positive pulses are essentially ineffective: for such pulses capacitive currents enter the axon more distally and over a much larger area, so that the resulting depolarization is slight.

The sheer number of direct responses strongly suggests that axons, not somata, are the main recipients of the stimuli, since geometry limits the number of somata that can be in close contact to a given electrode. Our results do not indicate whether the responses are recorded from axons or somata, but we believe they are from antidromically activated somata. The recorded pulse shapes resemble those from non-direct (synaptically mediated) responses. 


\subsection{Practical consequences}

We have determined a class of voltage-controlled stimulation waveforms that are effective at eliciting action potentials in nearby axons upon stimulation of a single substrate electrode. We were able to evoke activity, without ever requiring voltages that cause electrochemistry. By tuning the pulse amplitude, we could select how many cells to stimulate, since individual cells become responsive at sharply defi ned stimulus amplitudes. On about $20 \%$ of electrodes, tuning pulse amplitudes could even be used to select different subsets of cells to stimulate (Figure 7).

Surprisingly, we found that for almost all electrodes, positive-then-negative biphasic voltage-controlled stimuli are much more effective than either short or long negative current pulses that reached the same peak voltage. This is good news for long-term stimulation experiments, because voltage pulses can be explicitly designed to avoid electrochemistry, without having to monitor the results. We hope that this will encourage other researchers to explore the potential benefi ts of non-ionic stimulation using voltage-controlled pulses, not only in culture, but also in in-vivo situations.

Acknowledgments: This work was partially supported by grants N5044134-01A1 and NS38628 from NIH-NINDS, and EB000786 from NIH-NIBIB, and by the BurroughsWellcome Fund and the Whitaker Foundation. We thank our cell culture technician Sheri McKinney for very helpful assistance.

\section{Appendix: Identifying response components}

For each (stimulation electrode, recording electrode)-pair, we fi tted a mixture of Gaussians to the fi rst $30 \mathrm{~ms}$ of the peri-stimulus-time-histogram (PSTH), using a variation on the SMEM algorithm (Ueda et al., 2000) that automatically determines the appropriate number of Gaussian components in the mixture. Figure 9 shows an example of the input and output of the algorithm, which we dubbed FSMEM, for "Free Split/Merge Expectation-Maximalization".

We construct a histogram of latencies of spikes on a given recording channel in response to a given stimulation channel, then fi $t$ it with a probability density function of the form:

$$
p_{N, \vec{\mu}, \vec{\sigma}, \vec{\alpha}}(t)=\sum_{n=1}^{N} \frac{\alpha_{n}}{\sqrt{2 \pi} \sigma_{n}} e^{-\frac{\left(t-\mu_{n}\right)^{2}}{2 \sigma_{n}^{2}}} .
$$

Here $\mu_{n}$ are the centroids (in time) of each of the components, $\sigma_{n}$ are their widths, and $\alpha_{n}$ are the mixing coeffi cients. 


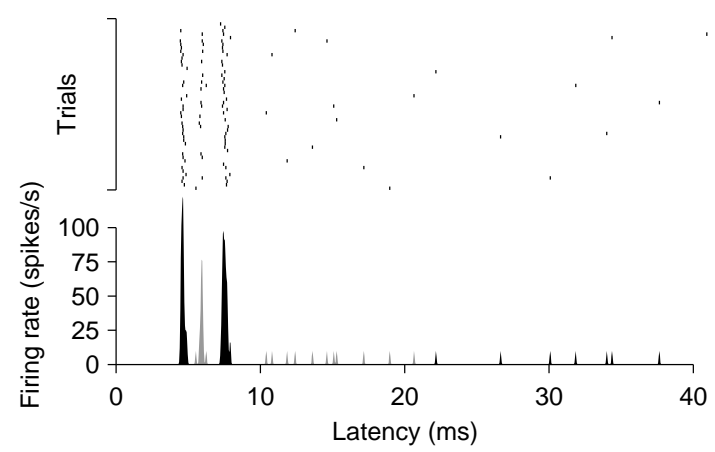

Figure 9. Classifying response components by FSMEM. Rasterplot of spikes recorded on one electrode in response to a distant electrode (top). FSMEM found five components (bottom) (alternate components are shown black and grey).

The fi $\mathrm{t}$ is performed by maximizing an objective function which is the sum of two terms:

$$
L=L_{0}+L_{\mathrm{MDL}}
$$

Here $L_{0}$ is the log-likelihood (Fisher, 1922),

$$
L_{0}=\sum_{i} \log p\left(t_{i}\right)
$$

(where $i$ sums over spikes, and $t_{i}$ is the latency of the $i$-th spike in the dataset). The other term is a minimum-description-length correction (Rissanen, 1978):

$$
L_{\mathrm{MDL}}=-(3 N-1)
$$

The inclusion of $L_{\mathrm{MDL}}$ allows the algorithm to autonomously select the number of components without bias.

After determining the distribution of responses, spikes were assigned to the most probable component in the histogram, so the reliability of each component could be determined in each stimulation condition.

The algorithm now consists of these steps:

(1) Start with $N=1$, and suitable default values for $\vec{\mu}$, $\vec{\sigma}$, and $\vec{\alpha}$.

(2) Optimize $\vec{\mu}$, $\vec{\sigma}$, and $\vec{\alpha}$ using expectation-maximalization (EM) (Dempster et al., 1977).

(3) For each component, $n$, consider whether splitting this component in two may improve the objective function $L=L_{0}+L_{\mathrm{MDL}}$ :

(a) Create a new cluster $N+1$ with $\mu_{N+1}=\mu_{n}+\alpha$ where $\alpha$ is a small random number. Set $\mu_{n} \leftarrow \mu_{n}-\alpha$. Set $\alpha_{N+1} \leftarrow \alpha_{n} / 2$, and then $\alpha_{n} \leftarrow \alpha_{n} / 2$. 
(b) Run EM on the subset of spikes associated with the original cluster $n$, operating only on the parameters of the new clusters $n$ and $N+1$.

(c) Run EM on the entire dataset.

(d) If this improves $L$, accept the split, otherwise reject it.

(4) If no split constitutes an improvement, consider whether merging any pair of clusters helps: For each pair:

(a) Replace cluster $n$ and $m$ by a single cluster $n$ with $\mu_{n} \leftarrow\left(\mu_{n}+\mu_{m}\right) / 2, \sigma_{n} \leftarrow$ $\left(\sigma_{n}+\sigma_{m}\right) / 2$, and $\alpha_{n} \leftarrow \alpha_{n}+\alpha_{m}$.

(b) Run EM on the subset of spikes associated with the original clusters $n$ and $m$, operating only on the parameters of the new cluster $n$.

(c) Run EM on the entire dataset.

(d) If this improves $L$, accept the split, otherwise reject it.

(5) If no merge constitutes an improvement, go back to considering merges.

(6) If neither splits nor merges improve $L$, the algorithm stops.

A more detailed description of the algorithm, as well as an implementation in Matlab (MathWorks), is available upon request.

\section{References}

Bai, Q., Wise, K. D., 2001. Single-unit neural recording with active microelectrode arrays. IEEE Trans. Biomed. Eng. 48 (8), 911-920.

Branner, A., Normann, R. A., 2000. A multielectrode array for intrafascicular recording and stimulation in sciatic nerve of cats. Brain Res. Bull. 51 (4), 293-306.

Buitenweg, J. R., Rutten, W. L. C., Willems, W. P. A., Nieuwkasteele, J. W. v., 1998. Measurement of sealing resistance of cell-electrode interfaces in neuronal cultures using impedance spectroscopy. Med. Biol. Eng. Comput. 36 (5), 630-637.

DeMarse, T. B., Wagenaar, D. A., Blau, A. W., Potter, S. M., 2001. The neurally controlled animat: biological brains acting with simulated bodies. Autonomous Robots 11, 305310 .

Dempster, A. P., Laird, N. M., Rubin, D. B., 1977. Maximum likelihood from incomplete data via EM algorithm. J. R. Stat. Soc. Ser. B-Methodol. 39 (1), 1-38.

Echevarria, D., Albus, K., 2000. Activity-dependent development of spontaneous bioelectric activity in organotypic cultures of rat occipital cortex. Dev. Brain Res. 123 (2), 151-164.

Egert, U., Schlosshauer, B., Fennrich, S., Nisch, W., Fejtl, M., Knott, T., Muller, T., Hammerle, H., 1998. A novel organotypic long-term culture of the rat hippocampus on substrate-integrated multielectrode arrays. Brain Research Protocols 2, 229-242.

Fisher, R., 1922. On the mathematical foundations of theoretical statistics. Philosophical Trans. of the Royal Society of London Series A 222, 309-368.

Gross, G. W., 1979. Simultaneous single unit recording in vitro with a photoetched laser deinsulated gold multimicroelectrode surface. IEEE Trans. Biomed. Eng. 26 (5), $273-$ 279. 
Gross, G. W., Rhoades, B. K., Reust, D. L., Schwalm, F. U., 1993. Stimulation of monolayer networks in culture through thin-fi $1 \mathrm{~m}$ indium-tin oxide recording electrodes. J. Neurosci. Meth. 50 (2), 131-143.

Grumet, A. E., Wyatt, Jr, J. L., Rizzo, III, J. F., 2000. Multi-electrode stimulation and recording in the isolated retina. J. Neurosci. Meth. 101 (1), 31-42.

Harsch, A., Robinson, H. P. C., 2000. Postsynaptic variability of fi ring in rat cortical neurons: The roles of input synchronization and synaptic nmda receptor conductance. J. Neurosci. 20 (16), 6181-6192.

Heck, D., 1995. Investigating dynamic aspects of brain-function in slice preparations spatiotemporal stimulus patterns generated with an easy-to-build multielectrode array. J. Neurosci. Meth. 58 (1-2), 81-87.

Heuschkel, M. O., Fejtl, M., Raggenbass, M., Bertrand, D., Renaud, P., 2002. A threedimensional multi-electrode array for multi-site stimulation and recording in acute brain slices. Journal of Neuroscience Methods 114 (2), 135-148.

Horowitz, P., Hill, W., 1996. The art of electronics. 2nd ed, Cambridge University press.

Jimbo, Y., Kawana, A., 1992. Electrical stimulation and recording from cultured neurons using a planar electrode array. Bioelectrochemistry and Bioenergetics 29, 193-204.

Jimbo, Y., Tateno, T., Robinson, H. P. C., 1999. Simultaneous induction of pathwayspecifi c potentiation and depression in networks of cortical neurons. Biophys. J. 76 (2), 670-678.

Kovacs, G. T. A., 1994. Introduction to the theory, design, and modeling of thin fi $\mathrm{lm}$ microdevices for neural interfaces. In: Enabling Technologies for Cultured Neural Networks. Academic Press, pp. 121-162.

Lilly, J. C., Hughes, J. R., Alvord, E. C., Galkin, T. W., 1955. Brief, noninjurious electric waveform for stimulation of the brain. Science 121 (3144), 468-469.

Maher, M. P., Dvorak-Carbone, H., Pine, J., Wright, J. A., Tai, Y.-C., 1999a. Microstructures for studies of cultured neural networks. Med. Biol. Eng. Comput. 37 (1), 110-118.

Maher, M. P., Pine, J., Wright, J., Tai, Y.-C., 1999b. The neurochip: a new multi-electrode device for stimulating and recording from cultured neurons. J. Neurosci. Meth. 87 (1), 45-56.

McAdams, E. T., Lackermeier, A., McLaughlin, J. A., Macken, D., 1995. The linear and non-linear electrical properties of the electrode-electrolyte interface. Biosens. Bioelectr. 10, 67-74.

McCreery, D. B., Agnew, W. F., Yuen, T. G. H., Bullara, L., 1990. Charge-density and charge per phase as cofactors in neural injury induced by electrical-stimulation. IEEE Trans. Biomed. Eng. 37 (10), 996-1001.

McIntyre, C. C., Grill, W. M., 1999. Excitation of central nervous system neurons by nonuniform electric fi elds. Biophys. J. 76 (2), 878-888.

McIntyre, C. C., Grill, W. M., 2001. Finite element analysis of the current-density and electric fi eld generated by metal microelectrodes. Ann. Biomed. Eng. 29 (3), 227-235.

Nakanishi, K., Kukita, F., 1998. Functional synapses in synchronized bursting of neocortical neurons in culture. Brain Res. 795 (1-2), 137-146.

Nicolelis, M. A. L., Ghazanfar, A. A., Stambaugh, C. R., Oliveira, L. M. O., Laubach, M., Chapin, J. K., Nelson, R. J., Kaas, J. H., 1998. Simultaneous encoding of tactile information by three primate cortical areas. Nat. Neurosci. 1 (7), 621-630. 
Novak, J. L., Wheeler, B. C., 1988. Multisite hippocampal slice recording and stimulation using a 32 element microelectrode array. J Neurosci Methods 23 (2), 149-59.

Pine, J., 1980. Recording action potentials from cultured neurons with extracellular microcircuit electrodes. J. Neurosci. Meth. 2 (1), 19-31.

Potter, S. M., 2001. Distributed processing in cultured neuronal networks. In: Nicolelis, M. A. L. (Ed.), Progress in Brain Research. Vol. 130. Elsevier Science, pp. 49-62.

Potter, S. M., DeMarse, T. B., 2001. A new approach to neural cell culture for long-term studies. J. Neurosci. Meth. 110 (1-2), 17-24.

Rattay, F., 1999. The basic mechanism for the electrical stimulation of the nervous system. Neuroscience 89 (2), 335-346.

Regehr, W. G., Pine, J., Rutledge, D. B., 1988. A long-term invitro silicon-based microelectrode-neuron connection. IEEE Trans. Biomed. Eng. 35 (12), 1023-1032.

Rissanen, J., 1978. Modeling by shortest data description. Automatica 14 (5), 465-471.

Shahaf, G., Marom, S., 2001. Learning in networks of cortical neurons. J. Neurosci. 21 (22), 8782-8788.

Shepherd, R. K., Matsushima, J., Millard, R. E., Clark, G. M., 1991. Cochlear pathology following chronic electrical-stimulation using noncharge balanced stimuli. Acta OtoLaryngol. 111 (5), 848-860.

Sigworth, F. J., Neher, E., 1980. Single Na+ channel currents observed in cultured rat muscle-cells. Nature 287 (5781), 447-449.

Thomas, Jr, C. A., Springer, P. A., Loeb, G. E., Berwald-Netter, Y., Okun, L. M., 1972. A miniature microelectrode array to monitor the bioelectric activity of cultured cells. Exp. Cell. Res. 74, 61-66.

Tscherter, A., Heuschkel, M. O., Renaud, P., Streit, J., 2001. Spatiotemporal characterization of rhythmic activity in rat spinal cord slice cultures. Eur. J. Neurosci. 14 (2), 179-190.

Ueda, N., Nakano, R., Ghahramani, Z., Hinton, G. E., 2000. SMEM algorithm for mixture models. Neural Comput. 12 (9), 2109-2128.

Wagenaar, D. A., Potter, S. M., 2002. Real-time multi-channel stimulus artifact suppression by local curve fi tting. J. Neurosci. Meth. 120 (2), 113-120.

Wagenaar, D. A., Potter, S. M., 2004. A versatile all-channel stimulator for electrode arrays, with real-time control. J. Neural Engineering (in press).

Weiland, J. D., Anderson, D. J., Humayun, M. S., 2002. In vitro electrical properties for iridium oxide versus titanium nitride stimulating electrodes. IEEE Trans. Biomed. Eng. 49 (12), 1574-1579.

Wise, K. D., Angell, J. B., 1975. Low-capacitance multielectrode probe for use in extracellular neurophysiology. IEEE Trans. Biomed. Eng. 22 (3), 212-219. 